\title{
Cytoplasmic dynein: a key player in neurodegenerative and neurodevelopmental diseases
}

\author{
CHEN Xiang-Jun ${ }^{1 \dagger}$, XU Huan ${ }^{1 \dagger}$, COOPER Helen $\mathrm{M}^{3}$ \& LIU Yaobo ${ }^{2 *}$ \\ ${ }^{1}$ Department of Neurology, Huashan Hospital, Fudan University, Shanghai 200040, China; \\ ${ }^{2}$ Institute of Neuroscience, Soochow University, Suzhou 215123, China; \\ ${ }^{3}$ Queensland Brain Institute, The University of Queensland, Brisbane, Queensland 4072, Australia
}

Received January 16, 2014; accepted February 25, 2014

\begin{abstract}
Cytoplasmic dynein is the most important molecular motor driving the movement of a wide range of cargoes towards the minus ends of microtubules. As a molecular motor protein, dynein performs a variety of basic cellular functions including organelle transport and centrosome assembly. In the nervous system, dynein has been demonstrated to be responsible for axonal retrograde transport. Many studies have revealed direct or indirect evidence of dynein in neurodegenerative diseases such as amyotrophic lateral sclerosis, Charcot-Marie-Tooth disease, Alzheimer's disease, Parkinson's disease and Huntington's disease. Among them, a number of mutant proteins involved in various neurodegenerative diseases interact with dynein. Axonal transport disruption is presented as a common feature occurring in neurodegenerative diseases. Dynein heavy chain mutant mice also show features of neurodegenerative diseases. Moreover, defects of dynein-dependent processes such as autophagy or clearance of aggregation-prone proteins are found in most of these diseases. Lines of evidence have also shown that dynein is associated with neurodevelopmental diseases. In this review, we focus on dynein involvement in different neurological diseases and discuss potential underlying mechanisms.
\end{abstract}

dynein, retrograde transport, pathogenesis, neurodegenerative diseases, neurodevelopmental diseases

Citation: Chen XJ, Xu H, Cooper HM, Liu YB. Cytoplasmic dynein: a key player in neurodegenerative and neurodevelopmental diseases. Sci China Life Sci, 2014, 57: 372-377, doi: 10.1007/s11427-014-4639-9

Mammalian cytoplasmic dynein was first identified and characterized as a high molecular weight microtubuleactivated ATPase and the major microtubule minus end-directed motor protein of the cell in 1987 [1]. It has also recently emerged as an important player in various neurological diseases.

Cytoplasmic dynein is a large complex, consisting of heavy chains (HCs, $530 \mathrm{kD}$ ), intermediate chains (ICs, $74 \mathrm{kD}$ ), light intermediate chains (LICs, 53-59 kD), and light chains (LCs, 10-14 kD). The HCs are encoded by only one gene, ICs, LICs and LCs are each encoded by two

$\dagger$ Contributed equally to this work

*Corresponding author (email: liuyaobo@suda.edu.cn) genes in vertebrates [2].

The precise structure of the intact complex is still unknown, but at its core lies a homodimer of heavy chains. The C-terminal region of this homodimer of the heavy chain is conserved and is the motor domain of the dynein complex involved in binding and hydrolysis of ATP for energy [3]. The N-terminal region of the dynein heavy chain is known as the stem domain and contains binding positions for LICs and ICs. LCs bind to the IC at separate sites. The ICs, LICs and LCs together, form the cargo binding domain of cytoplasmic dynein [4] (Figure 1).

Dynactin is a key subunit of the dynein complex. It is an important cofactor of the microtubule (MT) motor, with the dynactin $\mathrm{p} 150^{\mathrm{Glued}}$ subunit interacting directly with the ICs. 


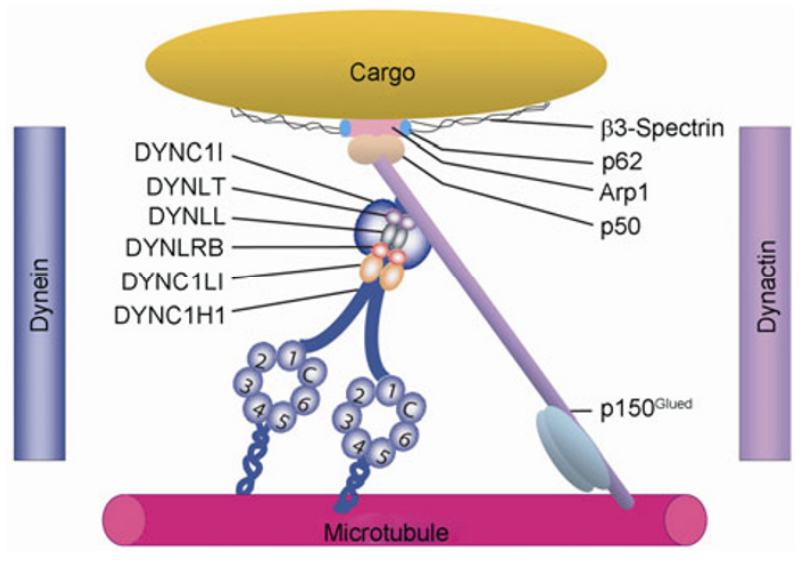

Figure 1 Structure of dynein-dynactin complex. Cartoon view of dynein-dynactin complex. The carboxy-terminal half of heavy chain subunits (DYNC1H1) are comprised by seven AAA-ATPase domains (labeled 1-6 and C). In amino terminal, the dynein intermediate chains (DYNC1I), light chains (DYNLT, DYNLL and DYNLRB), light-intermediate chains (DYNC1LI) and DYNC1H1 together form the cargo binding domain of dynein. Dynactin, which is a cofactor for the dynein, is itself a large protein complex with various subunits including p150 Glued, Arp1, p62 and p50. Cargo binding of dynein-dynactin complex requires the interactions of Arp1 and other proteins like $\beta 3$-Spectrin.

Dynactin also indirectly binds to the minus-end directed MTs as well as MT plus-end binding proteins [5,6]. It has been reported that the dynactin complex can enhance dynein's processivity without changing its rate of movement or ability to undergo retrograde transport $[7,8]$.

Cytoplasmic dynein plays crucial roles in cell survival, by mediating retrograde transport of various proteins/ protein complexes and membranous organelles. It is also important for cell division and cell differentiation $[2,3,8]$. The importance of dynein and its regulators is highlighted by their roles in the translocation of centrosomes along microtubules during mitosis, cell and nuclear migration and organelle transport [9-11]. The molecular interactions regulating dynein activity have recently been elucidated. It has been shown that lissencephaly 1 (LIS1), nuclear distribution protein E (NudE; also known as NdE), NudE-like (NudEL; also known as NdEL), Bicaudal D, ROD-ZW10-Zwilch (RZZ) and spindly are required for dynein function [12]. For example, these interactions play a crucial part in mitosis, including mitotic spindle orientation, nuclear and centrosomal transport in neuronal migration, centrosome positioning in non-neuronal cell migrating and growth cone extension [13].

Considerable evidence has shown that dysfunction of the dynein-dependent signaling pathway, and in particular, retrograde transport in neurons, results in various neurological diseases, exemplifying the clinical significance of dynein. This review will focus mainly on dynein-related neural diseases and present some possible mechanisms underlying the pathogenesis of these diseases.

\section{Dynein in neurodegenerative diseases}

Amyotrophic lateral sclerosis (ALS) is a fatal and progressive neurodegenerative disorder affecting motor neurons in the motor cortex, brainstem and spinal cord and typically leads to muscle weakness, muscle atrophy and death within about 5 years of onset. Sporadic ALS and autosomal dominant familial ALS (FALS) present with similar symptoms [14-16]. About $10 \%$ of ALS cases are inherited as an autosomal dominant trait, among which $15 \%-20 \%$ are found related to $\mathrm{Cu} / \mathrm{Zn}$ superoxide dismutase (SODI) gene mutations [17]. To date, more than 100 different mutations have been found in the SODI gene, and over 10 different genetic mouse models mimicking human MND pathology have been established [18] (http://ghr.nlm.nih.gov/gene/SOD1).

Swl, Cral and Loa are three different mutations in the dynein heavy chain. Loa and Cral are two different point mutations in Dynclhl. Swl is 9-bp deletion in Dynclhl that changes the four residues from position 1040-1043 into a single alanine, and it lies close to the Cral mutation. Lines of evidence have indicated that an involvement of dynein in SOD1 axonal transport deficit may be crucial in selective motor neuron degeneration in ALS. It was found that during embryonic development retrograde axonal transport defects were present in motor neurons of $S O D 1^{G 93 A}$ mice which has a significantly reduced life span. However, after crossing $S O D 1^{G 93 A}$ mice with Loa/+, double mutants Loa/SOD $1^{G 93 A}$ showed delayed disease progression and a significantly increased life span, with complete recovery in axonal transport deficits in motor neurons [19]. Cral/+ showed similar results when crossed with $S O D 1^{G 93 A}$ mice [20]. Studies have shown that SOD1 carrying ALS-linked mutations interacted and colocalized with the DYNC1H1 in vitro and in vivo [21]. It was proposed that the misfolded SOD1 mutants acquired an aberrant "gain-of-interaction" with dynein, thus overloading dynein-mediated retrograde transport and impairing axonal transport in motor neurons which ultimately led to motor neuron death [21]. However, mutant DYNC1H1 weakens this "gain-of-interaction", thus life span improvement in $\mathrm{Loa} / \mathrm{SOD} 1^{\mathrm{G} 93 \mathrm{~A}}$ mice may occur by rescuing the balance between anterograde and retrograde transport. Thus DYNC1H1 may ultimately ameliorate the loss of axonal homeostasis or rescue the imbalance of retrograde and anterograde axonal input [19,22].

Interestingly, our previous study has indicated that $\mathrm{Swl} /+$ mice harboring a nine-base pair deletion within exon 12 of Dynclhl, whose mutation located in dynein cargo binding domain, exhibit sensory neuropathy. More specifically, the $S w l$ mice only exhibited sensory neuropathy without motor neuronal deficits, while $\mathrm{Loa} /+$ and $\mathrm{Cral} /+$ mice mainly displayed motor neuropathy [23]. In contrast to Loa/+ mice, the $S w l /+$ mutation did not attenuate disease progression in $S O D 1^{G 93 A}$ mice in an intercross study [24]. This finding seems to contradict the study of Loa/SODI ${ }^{G 93 A}$ mice or 
Cral/SODI $1^{G 93 A}$ mice, but can be explained in two ways: firstly, different Dynclhl mutations could affect the affinity between dynein cargo binding domains and cargos. This would result in changes in the ability to transport cargos and cause different pathological severity. In $S w l /+$ mice, the nine-base pair deletion of Dynclhl may result in a small change in affinity of the dynein-dynactin complex for some cargos. This lower affinity may not be enough to induce observable changes in motor neurons but may do so in sensitive proprioceptive sensory neurons. Secondly, it is possible that different Dynclhl mutations may lead to selective cargo transport impairment thus induce selective neuronal degeneration [24].

Additional evidence has shown that mutant SOD1 can localize in mitochondria and disrupt mitochondria function [25]. As mitochondria and mutant SOD1 can both interact with dynein, it is possible that dynein-related accumulation of mutant SOD1 in mitochondria may lead to the direct impairment of mitochondrial function and the induction of neural degeneration. Dynein may also be important in SOD1 degradation as cells may utilize dynein-mediated transport to collect mutant SOD1 and form aggresomes for autophagic degradation [26]. Nevertheless, these findings still do not fully account for the pathogenesis of the selective neural degeneration seen in ALS, because the SOD1 mutation itself can only be found in some cases of FALS.

Other hypothesized mechanisms of dynein involvement in MND/ALS focus on the disturbance of retrograde transportation of some important cargos. Neurotrophic factors such as nerve growth factor (NGF), brain-derived neurotrophic factor (BDNF), and neurotrophin 3 (NT-3) are secreted by target tissues in early embryonic stages and subsequently bind to tyrosine kinase (Trk) receptors on the surface of neurons. Aberrant dynein complexes may undermine the retrograde transport of these endocytosed Trk receptors and downstream components to the soma, resulting in neural degeneration. However, some key questions remain, including which phase of disease progression is affected and which neurotrophins are required by individual neuron subtypes? This information is necessary to understand the role of Trk retrograde transport in selective neuron degeneration. Therefore, the pathological relationship between dynein and ALS still warrants further exploration.

\section{Charcot-Marie-Tooth (CMT) disease}

CMT is the most prevalent hereditary peripheral neuropathy affecting both motor and sensory nerves and has an estimated incidence of one in 2500 [27]. Clinically, it is characterized by distal limb muscle wasting and weakness, usually with skeletal deformities, distal sensory loss, and loss of deep tendon reflexes [28]. There are two main forms of CMT: demyelinating type 1 (CMT1) and axonal type 2 (CMT2) [29]. More than 40 loci and 30 genes have been identified in CMT (http://www.molgen.ua.ac.be/CMT-Mutations/ Mutations/MutByGene.cfm).

In a study of a CMT2 family with 23 members, three affected individuals were found to share a novel heterozygous variant DYNC1HIc.917A >G using exome sequencing method [30]. This mutation resulted in a missense substitution, p.His306Arg, at a highly conserved residue within the homodimerization domain. Affected patients typically have early-onset slowly progressive distal lower limb weakness and wasting with pes cavus deformity [30]. These patients shared some typical features with animal models of $S w l /+$, Loa/+ and Cral/+ that exhibit age-related progressive loss of muscle bulk and locomotor ability without a major reduction in life span.

The mechanisms underlying the CMT2 phenotype are still unknown, but increasing evidence has suggested that dynein is associated with this type of hereditary neuropathies. CMT2B is caused by the G-protein Rab7 mutation [31] and Rab7 controls dynein/dynactin recruitment to lysosomes and late endosomes through its effector protein RILP [32]. In CMT2E, mutant NF-L (neurofilament light) aggregates disturb microtubules and disrupt the normal localization of mitochondria [33]. Additionally, in the CMT2A mouse model, axonal transport of mitochondria is compromised [34]. Furthermore, the overexpression of CMT2Elinked NF-L leads to impaired axonal transport [35].

However, there is much debate about the exact roles of dynein in CMT disease. In 2003, Loa/+ was reported to affect motor neurons in the spinal anterior horn [36]. While our study suggested that Loa/ + mice also suffered from early-onset proprioceptive sensory neuropathy or possibly primary effects on the sensory neurons. A recent study by Wiggins [37], found that Loa/+ mice displayed deficits in both the motor and sensory nervous system. This argues against a model primarily involving sensory loss. This study found abnormal dendrites in large trigeminal motor neurons which is consistent with the findings in $\mathrm{Cral} /+$ mice [38]. The phenotype of the Loa/+ mouse differs considerably between the cranial and the spinal levels of the neuraxis. $\alpha$-motoneuron loss in the trigeminal motor nucleus was already apparent at an early postnatal age and progressed only minimally with age [37]. These findings give rise to questions of how dynein dysfunction results in selective neural degeneration, especially selective motor neuron degeneration. Which type of neuron is affected in CMT disease? What is the 'trigger' substance initiating CMT disease onset and progression?

Some clues suggest neurotrophic factors are involved in the pathogenesis of the disease. In fact, each factor functions during discrete developmental periods and target selection occurs at different time-points. For example, NT-3 signaling significantly affects neurons in the lumbar spinal cord, in contrast to those innervating the mastication muscles of the jaw $[37,39]$. Aberrant neurofilament accumulation in $\mathrm{Loa} /+$ and $\mathrm{Cral} /+$ mice may explain the motor neu- 
ron degeneration as being the result of dynein-mediated dysfunction of transport [40]. To date, many studies show a close relationship between dynein and CMT2 disease. However, the mechanisms of dynein-dependent pathology underlying CMT2 pathology in patients and related mouse models need further exploration.

\section{Alzheimer's disease (AD)}

Alzheimer's disease is the most common form of dementia and most often diagnosed in the elderly over 65 years of age. Alzheimer's disease has some general symptoms including progressive and irreversible cognitive deterioration with memory loss, impaired judgment and language, and other cognitive deficits and behavioral symptoms. Loss of body function can lead ultimately to death [41]. The typical pathological features are neurofibrillary tangles (NFTs) caused by tau protein deposits inside the neurons [42] and senile plaques (SPs). SPs are dense, mostly insoluble deposits of beta-amyloid peptide and cellular material outside and around the neuron [42]. The cause and progression of Alzheimer's disease are not well understood. APP is the precursor of $A \beta$ peptide and is transported by fast axonal anterograde transport [43]. A $\beta$ peptide has been shown to disrupt axonal transport in vitro [44]. Axonal transport is also compromised in aged brains [44]. Several lines of evidence investigating the link between axonal transport machinery and amyloid plaques suggested an involvement of anterograde transport rather than retrograde transport [45]. Studies of axonal transport revealed interactions between ADassociated proteins and dynein. In an AD animal model, aged cynomolgus monkeys, researchers found that $\mathrm{AD}$-associated proteins accumulated in the axon terminals and that DIC-DYN (dynactin) significantly increased along with axonal transport motor protein accumulation [46]. $R a b 5$, which has been reported to depend on dynein in endocytosis, stimulated up-regulation of the endocytic pathway and increased intracellular A $\beta$ production [47]. Furthermore, tau hyperphosphorylation has been observed in AD [48,49]. Somatic localization of phospho-tau, a cytoskeletal protein, is indicative of cytoskeletal breakdown [50]. A recent study indicates that altered cellular distribution of phospho-tau protein coincided with impaired retrograde axonal transport in neurons of aged rats, suggesting that failure of axonal trafficking may be responsible for the lack of neurotrophic support in aged cholinergic neurons of the basal forebrain [50]. It is also possible that tau hyperphosphorylation contributes to cytoskeletal instability, thereby impairing dynein's normal function. Perhaps dynein acts in association with tau and APP to promote AD progression. To date, most studies have shown an indirect relationship between dynein and AD. However direct evidence linking AD-related proteins and dynein is anticipated.

\section{Parkinson's disease (PD) and Huntington disease (HD)}

PD is one of the most common degenerative disorder of the central nervous system. Its typical symptoms are resting tremor, rigidity, slowness of movement, and postural instability. PD results from the loss of dopamine-generating cells in the substantia nigra. Mutations in Parkin, $\alpha$-synuclein, PINK1, and DJ1 are associated with PD. HD, another basal ganglia degenerative disease, has typical features including loss of muscle coordination, cognitive decline and psychiatric symptoms. These symptoms appear in mid-adult life. $\mathrm{HD}$ is caused by an expanded CAG repeat in exon 1 of the gene coding for the huntingtin protein [51]. Perry syndrome is an atypical PD syndrome resistant to L-DOPA, characterized by early-onset parkinsonism, depression, severe weight loss and hypoventilation, with brain pathology characterized by TDP-43 immunostaining [52]. There is some evidence that dynein plays an important part in PD/HD progression. Lewy bodies are abnormal protein aggregates that develop inside nerve cells in PD. In animal models, Lewy-like intracellular inclusion bodies in surviving neurons were observed in heterozygous $\mathrm{Cral} /+$ mice [36]. Additionally, Cral/+ mice display motor and behavioral abnormalities resembling HD and Perry syndrome [36]. These mice also display striatal atrophy and ventricle enlargement without striatal or substantia nigra dopaminergic neuronal loss. Dynein mutant striatal neurons also exhibit strongly disrupted neurite morphology in vitro. Additionally, genome-wide linkage analysis has identified diseasesegregating dynactin CAP-Gly domain substitutions that diminish microtubule binding and lead to intracytoplasmic inclusions [52]. Since dynactin is an important regulator of dynein, Perry syndrome may be closely related to the disruption of dynein function. However, we are a long way from fully understanding the mechanism by which dynein contributes to PD and HD.

\section{Dynein in neurodevelopmental diseases}

As a house keeping protein, dynein is not only important in neural degeneration, but is also crucial in neural development, including neurogenesis and neuronal migration.

LIS1 was reported to facilitate the activity of cytoplasmic dynein, interacting directly with the cytoplasmic dynein heavy chain (CDHC) and NudEL and its ortholog NudE. LIS1 and NudEL/NudE are important dynein interactors in the cytoplasmic dynein pathway [13]. Studies have shown that both LIS1 and NudEL are required for cell migration, nuclear, centrosomal, microtubule transport, mitosis, and growth cone motility [53-56]. It has been reported that the LIS1/ NudEL /dynein pathway is involved in neuronal migration and positioning during cortical development and is 
therefore essential for proper brain function [57]. The LISI gene is responsible for human lissencephaly (smooth brain), which is a neuronal migration disorder resulting from a failure or delay in neuronal migration and severe abnormalities in cortical layering [58]. LIS1 and NudEL are colocalized predominantly at the centrosome in early neuroblasts but are redistributed to axons via dynein. NudEL and LIS1 regulate the distribution of $\mathrm{CDHC}$ along microtubules and consequently affect $\mathrm{CDHC}$ activity during neuronal migration and axonal retrograde transport in a Cdk5/p35 (NudEL phosphorylator)-dependent manner [55]. As LIS1 and NudEL are regulators of dynein function, they may provide insights into important cell functions underlying neurodevelopmental diseases.

\section{Conclusion}

In summary, as a key retrograde transport protein, cytoplasmic dynein has increasingly attracted attention from researchers because of its important roles in retrograde axonal transport and its association with various neurological disorders. To date, studies have largely focused on dynein-cytoskeletal motor interactions and the link between disrupted dynein function and disease morphology. Therefore, further research is required to reveal the molecular mechanisms through which dynein subunits or dynein regulators trigger neurodegenerative disease and potentiate its progression. In conclusion, the mechanisms underlying molecular cross-talk between cytoplasmic dynein and dyneininteracting proteins warrant further study.

We thank the National Natural Science Foundation of China (81330026, 31271259, 30990261, 30871425 to Liu Yaobo; 30870873, 81171187 to Chen Xiang-Jun), the National Basic Research Program, Ministry of Science and Technology of China (2013CB945604), and the Chinese Academy of Sciences (KSCX2-EW-Q-11) for funding support. We also thank the Queensland Government's National and International Research Alliances Program and the National Health and Medical Research Council of Australia for financial support.

1 Paschal BM, Shpetner HS, Vallee RB. MAP 1C is a microtubule-activated ATPase which translocates microtubules in vitro and has dynein-like properties. J Cell Biol, 1987, 105: 1273-1282

2 Allan VJ. Cytoplasmic dynein. Biochem Soc Trans, 2011, 39: 1169-1178

3 Pfister KK, Shah PR, Hummerich H, Russ A, Cotton J, Annuar AA, King SM, Fisher EM. Genetic analysis of the cytoplasmic dynein subunit families. PLoS Genet, 2006, 2: e1

4 Pfister KK. Dynein cargo gets its groove back. Structure, 2005, 13: 172-173

5 Moughamian AJ, Holzbaur EL. Dynactin is required for transport initiation from the distal axon. Neuron, 2012, 74: 331-343

6 Vaughan KT, Vallee RB. Cytoplasmic dynein binds dynactin through a direct interaction between the intermediate chains and p150Glued. J Cell Biol, 1995, 131: 1507-1516

7 Kobayashi T, Shiroguchi K, Edamatsu M, Toyoshima YY. Microtubule-binding properties of dynactin p150 expedient for dynein motility. Biochem Biophys Res Commun, 2006, 340: 23-28
8 Culver-Hanlon TL, Lex SA, Stephens AD, Quintyne NJ, King SJ. A microtubule-binding domain in dynactin increases dynein processivity by skating along microtubules. Nat Cell Biol, 2006, 8: 264-270

9 Heald R, Tournebize R, Habermann A, Karsenti E, Hyman A. Spindle assembly in Xenopus egg extracts: respective roles of centrosomes and microtubule self-organization. J Cell Biol, 1997, 138: 615-628

10 Karki S, Holzbaur EL. Cytoplasmic dynein and dynactin in cell division and intracellular transport. Curr Opin Cell Biol, 1999, 11: 45-53

11 Vallee RB, Williams JC, Varma D, Barnhart LE. Dynein: an ancient motor protein involved in multiple modes of transport. J Neurobiol, 2004, 58: 189-200

12 Kardon JR, Vale RD. Regulators of the cytoplasmic dynein motor. Nat Rev Mol Cell Biol, 2009, 10: 854-865

13 McKenney RJ, Vershinin M, Kunwar A, Vallee RB, Gross SP. LIS1 and NudE induce a persistent dynein force-producing state. Cell, 2010, 141: 304-314

14 Chevalier-Larsen E, Holzbaur EL. Axonal transport and neurodegenerative disease. Biochim Biophys Acta, 2006, 1762: 1094-1108

15 Soo KY, Farg M, Atkin JD. Molecular motor proteins and amyotrophic lateral sclerosis. Int J Mol Sci, 2011, 12: 9057-9082

16 Rosen DR. Mutations in $\mathrm{Cu} / \mathrm{Zn}$ superoxide dismutase gene are associated with familial amyotrophic lateral sclerosis. Nature, 1993, 364: 362

17 Brown RJ. Amyotrophic lateral sclerosis: recent insights from genetics and transgenic mice. Cell, 1995, 80: 687-692

18 Turner BJ, Lopes EC, Cheema SS. Inducible superoxide dismutase 1 aggregation in transgenic amyotrophic lateral sclerosis mouse fibroblasts. J Cell Biochem, 2004, 91: 1074-1084

19 Kieran D, Hafezparast M, Bohnert S, Dick JR, Martin J, Schiavo G, Fisher EM, Greensmith L. A mutation in dynein rescues axonal transport defects and extends the life span of ALS mice. J Cell Biol, 2005, 169: 561-567

20 Teuchert M, Fischer D, Schwalenstoecker B, Habisch HJ, Bockers TM, Ludolph AC. A dynein mutation attenuates motor neuron degeneration in SOD1(G93A) mice. Exp Neurol, 2006, 198: 271-274

21 Zhang F, Strom AL, Fukada K, Lee S, Hayward LJ, Zhu H. Interaction between familial amyotrophic lateral sclerosis (ALS)-linked SOD1 mutants and the dynein complex. J Biol Chem, 2007, 282: 16691-16699

22 El-Kadi AM, Soura V, Hafezparast M. Defective axonal transport in motor neuron disease. J Neurosci Res, 2007, 85: 2557-2566

23 Banks GT, Fisher EM. Cytoplasmic dynein could be key to understanding neurodegeneration. Genome Biol, 2008, 9: 214

24 Chen XJ, Levedakou EN, Millen KJ, Wollmann RL, Soliven B, Popko B. Proprioceptive sensory neuropathy in mice with a mutation in the cytoplasmic Dynein heavy chain 1 gene. J Neurosci, 2007, 27: 14515-14524

25 Vande VC, Miller TM, Cashman NR, Cleveland DW. Selective association of misfolded ALS-linked mutant SOD1 with the cytoplasmic face of mitochondria. Proc Natl Acad Sci USA, 2008, 105: 4022-4027

26 Strom AL, Gal J, Shi P, Kasarskis EJ, Hayward LJ, Zhu H. Retrograde axonal transport and motor neuron disease. J Neurochem, 2008, 106: 495-505

27 Skre H. Genetic and clinical aspects of Charcot-Marie-Tooth's disease. Clin Genet, 1974, 6: 98-118

28 Pareyson D, Scaioli V, Laura M. Clinical and electrophysiological aspects of Charcot-Marie-Tooth disease. Neuromolecular Med, 2006, 8: 3-22

29 Szigeti K, Lupski JR. Charcot-Marie-Tooth disease. Eur J Hum Genet, 2009, 17: 703-710

30 Weedon MN, Hastings R, Caswell R, Xie W, Paszkiewicz K, Antoniadi $\mathrm{T}$, Williams $\mathrm{M}$, King $\mathrm{C}$, Greenhalgh $\mathrm{L}$, Newbury-Ecob R, Ellard S. Exome sequencing identifies a DYNC1H1 mutation in a large pedigree with dominant axonal Charcot-Marie-Tooth disease. Am J Hum Genet, 2011, 89: 308-312

31 Verhoeven K, De Jonghe P, Coen K, Verpoorten N, Auer-Grumbach M, Kwon JM, FitzPatrick D, Schmedding E, De Vriendt E, Jacobs A, 
Van Gerwen V, Wagner K, Hartung HP, Timmerman V. Mutations in the small GTP-ase late endosomal protein RAB7 cause Charcot-Marie-Tooth type 2B neuropathy. Am J Hum Genet, 2003, 72: $722-727$

32 Jordens I, Fernandez-Borja M, Marsman M, Dusseljee S, Janssen L, Calafat J, Janssen H, Wubbolts R, Neefjes J. The Rab7 effector protein RILP controls lysosomal transport by inducing the recruitment of dynein-dynactin motors. Curr Biol, 2001, 11: 1680-1685

33 Perez-Olle R, Jones ST, Liem RK. Phenotypic analysis of neurofilament light gene mutations linked to Charcot-Marie-Tooth disease in cell culture models. Hum Mol Genet, 2004, 13: 2207-2220

34 Misko A, Jiang S, Wegorzewska I, Milbrandt J, Baloh RH. Mitofusin 2 is necessary for transport of axonal mitochondria and interacts with the Miro/Milton complex. J Neurosci, 2010, 30: 4232-4240

35 Brownlees J, Ackerley S, Grierson AJ, Jacobsen NJ, Shea K, Anderton BH, Leigh PN, Shaw CE, Miller CC. Charcot-Marie-Tooth disease neurofilament mutations disrupt neurofilament assembly and axonal transport. Hum Mol Genet, 2002, 11: 2837-2844

36 Hafezparast M, Klocke R, Ruhrberg C, Marquardt A, Ahmad-Annuar A, Bowen S, Lalli G, Witherden AS, Hummerich H, Nicholson S, Morgan PJ, Oozageer R, Priestley JV, Averill S, King VR, Ball S, Peters J, Toda T, Yamamoto A, Hiraoka Y, Augustin M, Korthaus D, Wattler S, Wabnitz P, Dickneite C, Lampel S, Boehme F, Peraus G, Popp A, Rudelius M, Schlegel J, Fuchs H, Hrabe DAM, Schiavo G, Shima DT, Russ AP, Stumm G, Martin JE, Fisher EM. Mutations in dynein link motor neuron degeneration to defects in retrograde transport. Science, 2003, 300: 808-812

37 Wiggins LM, Kuta A, Stevens JC, Fisher EM, von Bartheld CS. A novel phenotype for the dynein heavy chain mutation Loa: altered dendritic morphology, organelle density, and reduced numbers of trigeminal motoneurons. J Comp Neurol, 2012, 520: 2757-2773

38 Zhang Z, Casey DM, Julien JP, Xu Z. Normal dendritic arborization in spinal motoneurons requires neurofilament subunit L. J Comp Neurol, 2002, 450: 144-152

39 Matsuo S, Ichikawa H, Silos-Santiago I, Arends JJ, Henderson TA, Kiyomiya K, Kurebe M, Jacquin MF. Proprioceptive afferents survive in the masseter muscle of trkC knockout mice. Neuroscience, 2000, 95: 209-216

40 Ding J, Allen E, Wang W, Valle A, Wu C, Nardine T, Cui B, Yi J, Taylor A, Jeon NL, Chu S, So Y, Vogel H, Tolwani R, Mobley W, Yang Y. Gene targeting of GAN in mouse causes a toxic accumulation of microtubule-associated protein 8 and impaired retrograde axonal transport. Hum Mol Genet, 2006, 15: 1451-463

41 Lopez-de-Ipina K, Alonso JB, Travieso CM, Sole-Casals J, Egiraun H, Faundez-Zanuy M, Ezeiza A, Barroso N, Ecay-Torres M, Martinez-Lage P, Martinez DLU. On the selection of non-invasive methods based on speech analysis oriented to automatic Alzheimer disease diagnosis. Sensors (Basel), 2013, 13: 6730-6745

42 Tiraboschi P, Hansen LA, Thal LJ, Corey-Bloom J. The importance of neuritic plaques and tangles to the development and evolution of AD. Neurology, 2004, 62: 1984-1989

43 Pigino G, Morfini G, Pelsman A, Mattson MP, Brady ST, Busciglio J. Alzheimer's presenilin 1 mutations impair kinesin-based axonal transport. J Neurosci, 2003, 23: 4499-4508

44 Pigino G, Morfini G, Atagi Y, Deshpande A, Yu C, Jungbauer L, LaDu M, Busciglio J, Brady S. Disruption of fast axonal transport is a pathogenic mechanism for intraneuronal amyloid beta. Proc Natl Acad Sci USA, 2009, 106: 5907-5912

45 Eschbach J, Dupuis L. Cytoplasmic dynein in neurodegeneration. Pharmacol Ther, 2011, 130: 348-363

46 Kimura N, Imamura O, Ono F, Terao K. Aging attenuates dynactin-dynein interaction: down-regulation of dynein causes accumulation of endogenous tau and amyloid precursor protein in human neuroblastoma cells. J Neurosci Res, 2007, 85: 2909-2916

47 Grbovic OM, Mathews PM, Jiang Y, Schmidt SD, Dinakar R, Summers-Terio NB, Ceresa BP, Nixon RA, Cataldo AM. Rab5-stimulated up-regulation of the endocytic pathway increases intracellular beta-cleaved amyloid precursor protein carboxyl-terminal fragment levels and Abeta production. J Biol Chem, 2003, 278: 31261-31268

48 Zhao H, Chang R, Che H, Wang J, Yang L, Fang W, Xia Y, Li N, Ma Q, Wang X. Hyperphosphorylation of tau protein by calpain regulation in retina of Alzheimer's disease transgenic mouse. Neurosci Lett, 2013, 551: 12-16

49 Geekiyanage H, Upadhye A, Chan C. Inhibition of serine palmitoyltransferase reduces Abeta and tau hyperphosphorylation in a murine model: a safe therapeutic strategy for Alzheimer's disease. Neurobiol Aging, 2013, 34: 2037-2051

50 Niewiadomska G, Baksalerska-Pazera M, Riedel G. Altered cellular distribution of phospho-tau proteins coincides with impaired retrograde axonal transport in neurons of aged rats. Ann N Y Acad Sci, 2005, 1048: 287-295

51 Petersen A, Larsen KE, Behr GG, Romero N, Przedborski S, Brundin P, Sulzer D. Expanded CAG repeats in exon 1 of the Huntington's disease gene stimulate dopamine-mediated striatal neuron autophagy and degeneration. Hum Mol Genet, 2001, 10: 1243-1254

52 Farrer MJ, Hulihan MM, Kachergus JM, Dachsel JC, Stoessl AJ, Grantier LL, Calne S, Calne DB, Lechevalier B, Chapon F, Tsuboi Y, Yamada T, Gutmann L, Elibol B, Bhatia KP, Wider C, Vilarino-Guell C, Ross OA, Brown LA, Castanedes-Casey M, Dickson DW, Wszolek ZK. DCTN1 mutations in Perry syndrome. Nat Genet, 2009, 41: 163-165

53 McKenney RJ, Vershinin M, Kunwar A, Vallee RB, Gross SP. LIS1 and NudE induce a persistent dynein force-producing state. Cell, 2010, 141: 304-314

54 Grabham PW, Seale GE, Bennecib M, Goldberg DJ, Vallee RB. Cytoplasmic dynein and LIS1 are required for microtubule advance during growth cone remodeling and fast axonal outgrowth. J Neurosci, 2007, 27: 5823-5834

55 Sasaki S, Shionoya A, Ishida M, Gambello MJ, Yingling J, Wynshaw-Boris A, Hirotsune S. A LIS1/NUDEL/cytoplasmic dynein heavy chain complex in the developing and adult nervous system. Neuron, 2000, 28: 681-696

56 Shu T, Ayala R, Nguyen MD, Xie Z, Gleeson JG, Tsai LH. Ndel1 operates in a common pathway with LIS1 and cytoplasmic dynein to regulate cortical neuronal positioning. Neuron, 2004, 44: 263-277

57 Shu T, Ayala R, Nguyen MD, Xie Z, Gleeson JG, Tsai LH. Ndel1 operates in a common pathway with LIS1 and cytoplasmic dynein to regulate cortical neuronal positioning. Neuron, 2004, 44: 263-277

58 Moon HM, Wynshaw-Boris A. Cytoskeleton in action: lissencephaly, a neuronal migration disorder. Wiley Interdiscip Rev Dev Biol, 2013, 2: $229-245$

Open Access This article is distributed under the terms of the Creative Commons Attribution License which permits any use, distribution, and reproduction in any medium, provided the original author(s) and source are credited. 